\title{
Immunization against the Agent of Human Granulocytic Ehrlichiosis in a Murine Model
}

\author{
Wei Sun, ${ }^{\star}$ Jacob W. IJdo, ${ }^{\star}$ Sam R. Telford III, ${ }^{\S}$ Emir Hodzic, ${ }^{\ddagger}$ Yan Zhang, ${ }^{\star}$ Stephen W. Barthold, ${ }^{\ddagger}$ and Erol Fikrig ${ }^{\star}$ \\ $*$ Section of Rheumatology, Department of Internal Medicine, and ${ }^{\ddagger}$ Section of Comparative Medicine, Yale University School of Medicine, \\ New Haven, Connecticut 06520; and ${ }^{\S}$ Department of Tropical Public Health, Harvard University School of Public Health, Boston, \\ Massachusetts 02115
}

\begin{abstract}
The agent of human granulocytic ehrlichiosis (HGE) is a newly recognized tick-borne pathogen that resides within polymorphonuclear leukocytes. $\mathrm{C} 3 \mathrm{H} / \mathrm{HeN}$ mice can become infected with the agent of HGE (designated aoHGE) by syringe inoculation or tick-borne infection and develop transient neutropenia. They thereby partially mimic human disease and provide a model in which to study immunity to this microorganism. Mice vaccinated with lysates of purified aoHGE, or administered aoHGE antisera, were partially protected from both syringe- and tick-transmitted challenge with aoHGE. These data suggest that antibodies are sufficient to provide substantial, but not complete, immunity against aoHGE. (J. Clin. Invest. 1997. 100:30143018.) Key words: vaccine $\cdot$ Ehrlichia $\bullet$ antibody $\bullet$ ticks • intracellular pathogen
\end{abstract}

\section{Introduction}

Human granulocytic ehrlichiosis $(\mathrm{HGE})^{1}$ is an emerging tickborne infectious disease, and the pathogen infects and survives within granulocytes (1-6). Patients may develop fever, myalgia, and neutropenia, among other symptoms. Severe infection can be fatal, usually due to secondary infections $(3,5,7,8)$. Horses, sheep, goats, and humans acquire granulocytic ehrlichiosis, and the respective agents, Ehrlichia equi, Ehrlichia phagocytophila, and the agent of HGE (designated aoHGE) are genetically similar (9-11). Peromyscus leucopus, the whitefooted mouse, is an animal reservoir for aoHGE, and Ixodes scapularis (also known as Ixodes dammini) is an arthropod vector of aoHGE $(6,12,13)$. Not surprisingly, HGE is prevalent in the upper midwest and northeast United States, areas

W. Sun and J. IJdo contributed equally to this work.

Address correspondence to Erol Fikrig, M.D., Section of Rheumatology, Department of Internal Medicine, Yale University School of Medicine, 610 Laboratory of Clinical Investigation, 333 Cedar Street, New Haven, CT 06520-8031. Phone: 203-785-2454; FAX: 203785-7053; E-mail: erol.fikrig@yale.edu

Received for publication 28 May 1997 and accepted in revised form 1 October 1997.

1. Abbreviations used in this paper: aoHGE, agent of human granulocytic ehrlichiosis; HGE, human granulocytic ehrlichiosis.

J. Clin. Invest.

(C) The American Society for Clinical Investigation, Inc. 0021-9738/97/12/3014/05 \$2.00

Volume 100, Number 12, December 1997, 3014-3018

http://www.jci.org where other I. scapularis-borne microorganisms, including Borrelia burgdorferi and Babesia microti, are common (2, 5, 14). Laboratory mice have been infected with aoHGE (13). We now show that $\mathrm{C} 3 \mathrm{H} / \mathrm{HeN}$ mice are susceptible to aoHGE infection and also develop neutropenia, thereby partially resembling human infection. Moreover, when examined at $14 \mathrm{~d}$ after aoHGE challenge, mice vaccinated with aoHGE lysates are substantially protected from ehrlichiosis, and aoHGE-specific antibodies are sufficient to provide partial immunity from tick-borne aoHGE infection. These data provide a basis for beginning to understand protective immunity to this unique emerging pathogen.

\section{Methods}

Mice. 3-wk-old female $\mathrm{C} 3 \mathrm{H} / \mathrm{HeNCr}$ mice were obtained from the Frederick Cancer Research Center (Frederick, MD). C3H/HeJ mice, which are LPS unresponsive, have been shown to be susceptible to aoHGE infection (13). We chose $\mathrm{C} 3 \mathrm{H} / \mathrm{HeNCr}(\mathrm{C} 3 \mathrm{H})$ mice because they are LPS responsive and therefore more capable of responding to bacterial cell wall products (15). Mice were housed in filter-frame cages and killed with $\mathrm{CO}_{2}$. $\mathrm{C} 3 \mathrm{H}$ mice were initially inoculated intraperitoneally with blood $(50 \mu \mathrm{l})$ from $\mathrm{CD}-1$ mice that had been infected with the NCH-1 isolate (see purification of aoHGE and tickborne aoHGE vaccination studies) 3 wk earlier. In subsequent studies, $\mathrm{C} 3 \mathrm{H}$ mice were engorged upon by aoHGE-infected ticks.

Purification of aoHGE. aoHGE lysate was made from the NCH-1 isolate, which was recovered from the peripheral blood of a patient from Nantucket, MA (16). The NCH-1 isolate (passage 2) used for purification of aoHGE was the same organism used in subsequent needle- or tick-challenge studies. The $\mathrm{NCH}-1$ isolate was cultured using the HL-60 (240-CCL; American Type Culture Collection, Rockville, MD) cell line in Iscove's modified Dulbecco's medium supplemented with $20 \%$ FCS, using previously described methodologies (17). Cultures were maintained at $37^{\circ} \mathrm{C}$ in $5 \% \mathrm{CO}_{2}$. Fresh cells and medium were added biweekly to maintain a density of $\sim 10^{6}$ cells $/ \mathrm{ml}$. To ensure that the HL-60 cells were infected with aoHGE, cell cultures were placed onto microscope slides, stained with DiffQuick (Baxter Healthcare Corp., Miami, FL), and examined by light microscopy.

To purify aoHGE lysates, 1,000 ml of aoHGE-infected HL-60 cells were centrifuged at $1,500 \mathrm{rpm}$ for $10 \mathrm{~min}$, the pellet was resuspended with PBS-glucose $(0.02 \%)$, and centrifuged again using the same conditions. The resuspended cells were disrupted by shearing using 21gauge needles and pelleted. The supernatant was then digested with DNase/RNase $(50 \mu \mathrm{g} / \mathrm{ml})$ and subjected to density gradient ultracentrifugation using 42 and $30 \%$ discontinuous gradient renografin $(\mathrm{Ny}-$ comed Princeton, NJ) (18). Centrifugation was performed at 22,000 rpm for $75 \mathrm{~min}$. Ehrlichia bacteria were collected in a band at the 30 and $42 \%$ renografin interface.

Tick-borne aoHGE vaccination studies. I. scapularis larvae were allowed to feed to repletion on CD-1 mice that had been infected with the aoHGE NCH-1 isolate for $3 \mathrm{wk}$. $\mathrm{C} 3 \mathrm{H}$ mice could also have been used for this purpose. However, both $\mathrm{CD}-1$ and $\mathrm{C} 3 \mathrm{H}$ mice can be infected with aoHGE, and CD-1 mice are less expensive and have been used for aoHGE tick infestation for $>1 \mathrm{yr}$ by our group. The 
goal of this portion of the protocol was to obtain aoHGE-infected ticks rather than examine ehrlichiosis in the mice. The engorged larvae were collected, and kept until they had molted to nymphs. A sample was then examined for aoHGE infection by visual inspection of the salivary glands using the Feulgen reaction (13). The ticks had an $85 \%$ aoHGE infection rate.

For the vaccination studies, three or four aoHGE-infected I. scapularis nymphs were then placed, and allowed to engorge to repletion, on individual $\mathrm{C} 3 \mathrm{H}$ mice that were immunized with either aoHGE lysates in CFA or CFA alone (control). aoHGE lysate was dialyzed against PBS, and heat-treated at $56^{\circ} \mathrm{C}$ for $1 \mathrm{~h}$, before immunization. Groups of three to five mice were immunized subcutaneously in the back with $15 \mu \mathrm{g}$ of purified, heat-killed aoHGE lysates in CFA and boosted twice with the same preparation in incomplete Freund's adjuvant at bimonthly intervals.

$14 \mathrm{~d}$ after the ticks had fallen from the immunized animals, the mice were killed with $\mathrm{CO}_{2}$ and blood was obtained by cardiac exsanguination. To visualize morulae, blood smears were air-dried, and stained with DiffQuick. In each smear, 200 high power fields were examined for morulae. The presence of one or more definitive morulae was considered positive, and the percentage of aoHGE-infected neutrophils was calculated. At necropsy, $100 \mu \mathrm{l}$ of anticoagulated blood from each mouse was inoculated into culture flasks containing $5 \mathrm{ml}$ of $5 \times 10^{5}$ to $1 \times 10^{6} \mathrm{HL}-60$ cells $/ \mathrm{ml}$. aoHGE infection of the HL- 60 cells was determined at weeks 2, 3, 4, and 6. Mice were examined for aoHGE by PCR as described (see PCR and quantitative PCR).

$P C R$. An aoHGE-specific 16S rDNA fragment was amplified by PCR from peripheral blood. $50 \mu$ l of blood was lysed in red cell lysis buffer $\left(155 \mathrm{mM} \mathrm{NH}_{4} \mathrm{Cl} ; 10 \mathrm{mM} \mathrm{KHCO}_{3} ; 1 \mathrm{mM}\right.$ EDTA) and then treated with $10 \mu \mathrm{g} / \mu \mathrm{l}$ proteinase $\mathrm{K}$ at $56^{\circ} \mathrm{C}$ for $1 \mathrm{~h} .5 \mu \mathrm{l}$ of the supernatant containing aoHGE was used as template. Oligonucleotide primers were 497-521 (5'-TGT AGG CGG TTC GGT AAG TTA AAG-3') and 747-727 (5'-GCA CTC ATC GTT TAC AGC GTG-3'), which amplify a region that distinguishes aoHGE from other ehrlichia (6). PCR was done for 35 cycles with the following denaturation, annealing, and extension conditions: $1 \mathrm{~min}$ at $94^{\circ} \mathrm{C}, 1 \mathrm{~min}$ at $54^{\circ} \mathrm{C}$, and 2 min at $72^{\circ} \mathrm{C}$. PCR fragments were separated in a $1 \%$ agarose gel and stained with ethidium bromide or then transferred to nitrocellulose for Southern blotting using an aoHGE $16 \mathrm{rDNA}$ probe. In all of the PCR studies, blood from three to five individual naive (uninfected) mice was also used as a control template.

Quantitative PCR. To determine the detection limits of the PCR assay, serial 10-fold dilutions of purified aoHGE DNA was subjected to amplification. $5 \times 10^{-10} \mathrm{~g}$ (1:1,000 dilution of $\left.500 \mathrm{ng}\right)$ of purified aoHGE DNA was used as the initial template and an amplified product could be detected when up to $5 \times 10^{-16} \mathrm{~g}$ of aoHGE DNA was used as the template. Pulse-field gel electrophoresis demonstrated that aoHGE has a chromosome that migrates at $\sim 700 \mathrm{~kb}$ (not shown). We can estimate that $1 \mathrm{~mol}$ of aoHGE had a molecular mass of $\sim 4.6 \times$ $10^{8} \mathrm{~g}\left(7.0 \times 10^{5} \mathrm{bp} \times 660 \mathrm{~g} / \mathrm{bp}\right) .1$ aoHGE therefore has an approximate molecular mass of $7.6 \times 10^{-16} \mathrm{~g}\left(\left[4.6 \times 10^{8} \mathrm{~g} / \mathrm{mol}\right] / 6.02 \times 10^{23}\right.$ organisms $/ \mathrm{mol}$ ). Thus, the limits of detection of the PCR assay is estimated at nearly one organism. In addition, the sensitivity of the PCR was unchanged when purified aoHGE DNA was added to $50 \mu \mathrm{l}$ of murine blood and then subjected to red cell lysis buffer and proteinase K treatment (see PCR) before being used as the template, the same conditions as blood from experimental animals.

Passive antibody transfer studies. aoHGE antisera were obtained from $\mathrm{C} 3 \mathrm{H}$ mice subcutaneously immunized with $15 \mu \mathrm{g}$ of aoHGE lysate in CFA and boosted twice bimonthly with the same antigen in incomplete Freund's adjuvant. 2 wk after the final boost, blood was obtained by cardiac exsanguination. For the passive immunization studies, aoHGE antisera were diluted 1:5 in PBS and intradermally injected $(200 \mu \mathrm{l})$ into naive mice. $1 \mathrm{~d}$ after immunization, the mice were inoculated intraperitoneally with blood $(50 \mu \mathrm{l})$ from mice that had been infected with the NCH-1 isolate 2 wk earlier, or were engorged upon by three or four aoHGE-infected ticks. Mice were then administered the same amount of antisera (1:5 dilution in PBS, $200 \mu \mathrm{l})$ on days 4,8 , and 12 , and then killed on day 14 , and examined for infection. In these studies, a $100-\mu l$ aliquot of anticoagulated blood was evaluated for neutropenia using a Coulter counter (Antech Diagnostics, Farmingdale, NY). For the assessment of splenomegaly, the whole spleen was removed from each animal at necropsy and immediately weighed.

Immunoblot. $500 \mathrm{ng}$ of purified aoHGE lysate was separated using $15 \%$ SDS-PAGE and transferred onto nitrocellulose membranes. Membrane strips were incubated with sera $(1: 1,000,1: 10,000$, and 1:50,000 dilutions) from aoHGE-immunized mice or control mice (immunized with CFA) at room temperature for $1 \mathrm{~h}$, washed three times with PBS, and then incubated with alkaline phosphatase-conjugated anti-mouse IgG (Sigma Chemical Co., St. Louis, MO). The color was developed using BCIP/NBT (Kirkegaard and Perry, Gaithersburg, MD) as the substrate.

\section{Results and Discussion}

We first show that all five $\mathrm{C} 3 \mathrm{H}$ mice intraperitoneally inoculated with aoHGE developed transient neutropenia, with cytoplasmic inclusions known as morulae in $8-10 \%$ of the polymorphonuclear leukocytes at $2 \mathrm{wk}$. The aoHGE isolate (NCH-1) used in these studies was recovered previously from a woman with fever, neutropenia, and morulae in peripheral polymorphonuclear leukocytes (16). The average number of neutrophils in five aoHGE-infected mice at $14 \mathrm{~d}\left(814 \mathrm{cells} / \mathrm{mm}^{3}\right.$, $\pm 213 \mathrm{SD})$ was much lower than in five control animals $(3,421$ cells $\left./ \mathrm{mm}^{3}, \pm 426 \mathrm{SD}\right)$ that were inoculated with normal mouse blood. aoHGE-infected mice also developed splenomegaly $(0.32 \mathrm{~g}, \pm 0.05 \mathrm{SD})$ compared with controls $(0.14 \mathrm{~g}, \pm 0.02 \mathrm{SD})$. Neutropenia resolved by $60 \mathrm{~d}$ and morulae were no longer readily detectable in peripheral smears at this time point. This experimental murine model partially resembles human infection and can therefore be used to study pathogenesis and immunity.

Studies were performed to determine whether mice could be protected against aoHGE. Indeed, infection of a horse with aoHGE has been shown to confer resistance to challenge with E. equi (19). Groups of mice were actively immunized with purified aoHGE lysates. $2 \mathrm{wk}$ after the final boost, mice developed antibodies to aoHGE, detectable at a dilution of up to 1:10,000 on immunoblot. Antisera reacted predominantly against 40-, 44-, 65-, and 80-kD aoHGE antigens, among others (Fig. 1). Control mice, immunized with adjuvant in an identical fashion, did not develop aoHGE antibodies.

aoHGE-vaccinated mice were challenged with aoHGE, killed at $2 \mathrm{wk}$, and examined for infection (Table I). To simu-

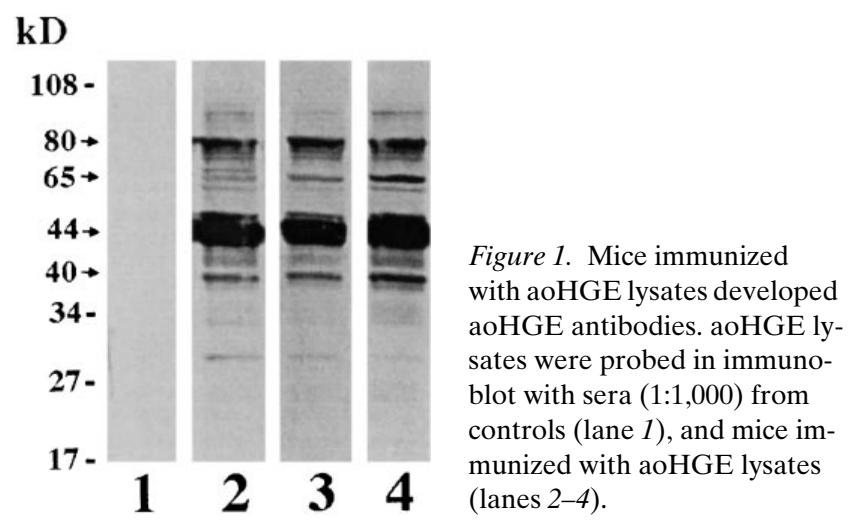


Table I. Mice Immunized with aoHGE Lysates Were Protected from Tick-borne Infection with aoHGE at $14 d$ after Challenge

\begin{tabular}{|c|c|c|c|c|}
\hline Immunogen & Mouse & Culture & Morulae & PCR \\
\hline \multicolumn{5}{|l|}{ Experiment 1} \\
\hline \multirow[t]{4}{*}{ CFA, control } & 1 & + & + & + \\
\hline & 2 & + & - & + \\
\hline & 3 & + & + & + \\
\hline & 4 & + & + & + \\
\hline \multirow[t]{4}{*}{ aoHGE lysates } & 5 & - & - & - \\
\hline & 6 & - & - & + \\
\hline & 7 & - & - & + \\
\hline & 8 & - & - & - \\
\hline \multicolumn{5}{|l|}{ Experiment 2} \\
\hline \multirow[t]{5}{*}{ CFA, control } & 1 & + & + & + \\
\hline & 2 & + & + & + \\
\hline & 3 & - & - & + \\
\hline & 4 & - & - & + \\
\hline & 5 & - & - & + \\
\hline \multirow[t]{5}{*}{ aoHGE lysates } & 6 & - & - & + \\
\hline & 7 & - & - & + \\
\hline & 8 & - & - & - \\
\hline & 9 & - & - & + \\
\hline & 10 & - & - & - \\
\hline
\end{tabular}

Peripheral smears were examined (200 granulocytes per smear) for aoHGE morulae within neutrophils. The presence of one or more definitive morulae was considered positive for aoHGE infection. For cultivation of aoHGE, $100 \mu \mathrm{l}$ of murine blood was inoculated into a culture of HL-60 cells. The cells were maintained for $6 \mathrm{wk}$ and examined for aoHGE infection. PCR was performed using 16S rDNA primers and $5 \mu \mathrm{l}$ of blood as the template.

late the natural mode of transmission, mice were engorged upon by nymphal ticks that had been infected with the NCH-1 isolate (Table I). Mice were assessed for infection at $14 \mathrm{~d}$ after tick feeding by examination of peripheral blood smears for morulae, culture, and by $16 \mathrm{~S}$ rDNA PCR of blood. Five of nine control animals had morulae in peripheral polymorphonuclear leukocytes, six of nine mice were culture positive, and aoHGE-specific DNA was detected in blood of all control mice (Table I). In contrast, morulae were not detected in blood smears of aoHGE-vaccinated mice $\left(\chi^{2}, P<0.01\right.$, compared with control mice) and aoHGE was not cultured from any of the nine mice $\left(\chi^{2}, P<0.005\right.$, compared with control mice), but five of nine animals were PCR positive $\left(\chi^{2}, P<0.01\right.$, compared with control mice). An amplified DNA product was not detected in samples from naive, uninfected mice, validating the specificity of the PCR assays. PCR reactions in which distilled water was used as the template were also uniformly negative. In addition, a DNA product was not obtained when blood from a mouse that had been immunized with aoHGE lysates in CFA (obtained $14 \mathrm{~d}$ after immunization) was used as the template, indicating that DNA from the vaccination procedure does not result in false-positive PCR reactivity.

Studies were then performed to determine whether antibodies were sufficient for protection. In three separate experiments, groups of three to five mice were passively immunized with aoHGE antisera, then challenged by either ticks or by direct inoculation of whole blood from an aoHGE-infected mouse (Table II). Regardless of the mode of aoHGE challenge, passive immunization of mice with aoHGE antisera was sufficient to afford substantial protection as determined by the lack of visualization of morulae in polymorphonuclear leukocytes on peripheral blood smears (7 of 11 control animals, 1 of 12 immunized mice: $\left.\chi^{2}, P<0.005\right)$, lack of recovery of aoHGE by culture ( 7 of 11 control animals, 1 of 12 immunized mice: $\chi^{2}$, $P<0.005)$, and the incomplete ability to amplify aoHGE DNA from blood by PCR (10 of 11 control animals, 4 of $12 \mathrm{immu-}$ nized mice: $\left.\chi^{2}, P<0.01\right)$. Furthermore, neutropenia was evident in aoHGE-infected control mice (462 cells $/ \mathrm{mm}^{3}, \pm 280$ SD) compared with uninfected animals $\left(3,240\right.$ cells $/ \mathrm{mm}^{3}$, $\pm 1,340 \mathrm{SD})$. Splenomegaly was also observed in aoHGEinfected control animals $(0.27 \mathrm{~g}, \pm 0.05 \mathrm{SD})$ but not in experimental mice $(0.12 \mathrm{~g}, \pm 0.03 \mathrm{SD})$.

Moreover, serial dilution PCR analysis indicated that the amount of aoHGE DNA was much lower in the PCR-positive, immunized mice compared with PCR-positive, control animals. Serial 10-fold dilutions of an aliquot of purified aoHGE DNA estimated that PCR could detect as little as one organism, indicating that the assay is sensitive. A product could be identified in PCR-positive, control mice at a serum dilution of $10^{5}-10^{8}$, whereas amplified DNA was discernible in the PCRpositive, vaccinated mice at a dilution of $10^{0}-10^{3}$ (Fig. 2 , as an example of one experiment). This suggested two possibilities.

Table II. Mice Passively Administered aoHGE Antisera Were Protected from either Tick-transmitted or Syringe-mediated Infection with aoHGE at $14 d$ after Challenge

\begin{tabular}{|c|c|c|c|c|}
\hline Immunogen (aoHGE challenge) & Mouse & Culture & Morulae & PCR \\
\hline \multicolumn{5}{|l|}{ Experiment 1 (tick-borne) } \\
\hline \multirow[t]{3}{*}{ NMS, control } & 1 & - & - & - \\
\hline & 2 & + & + & + \\
\hline & 3 & + & + & + \\
\hline \multirow[t]{3}{*}{ aoHGE antisera } & 4 & - & - & - \\
\hline & 5 & - & - & - \\
\hline & 6 & - & - & - \\
\hline \multicolumn{5}{|l|}{ Experiment 2 (tick-borne) } \\
\hline \multirow[t]{5}{*}{ NMS, control } & 1 & + & + & + \\
\hline & 2 & + & + & + \\
\hline & 3 & - & - & + \\
\hline & 4 & - & - & + \\
\hline & 5 & - & - & + \\
\hline \multirow[t]{5}{*}{ aoHGE antisera } & 6 & - & - & - \\
\hline & 7 & - & - & - \\
\hline & 8 & + & + & + \\
\hline & 9 & - & - & + \\
\hline & 10 & - & - & + \\
\hline \multicolumn{5}{|l|}{ Experiment 3 (syringe-inocula) } \\
\hline \multirow[t]{3}{*}{ NMS, control } & 1 & + & + & + \\
\hline & 2 & + & + & + \\
\hline & 3 & + & + & + \\
\hline \multirow[t]{4}{*}{ aoHGE antisera } & 4 & - & - & - \\
\hline & 5 & $\mathrm{C}$ & - & - \\
\hline & 6 & $\mathrm{C}$ & - & - \\
\hline & 7 & - & - & + \\
\hline
\end{tabular}

Peripheral smears, culture, and PCR were assessed as in Table I. NMS, Normal mouse sera; $C$, contaminated. 

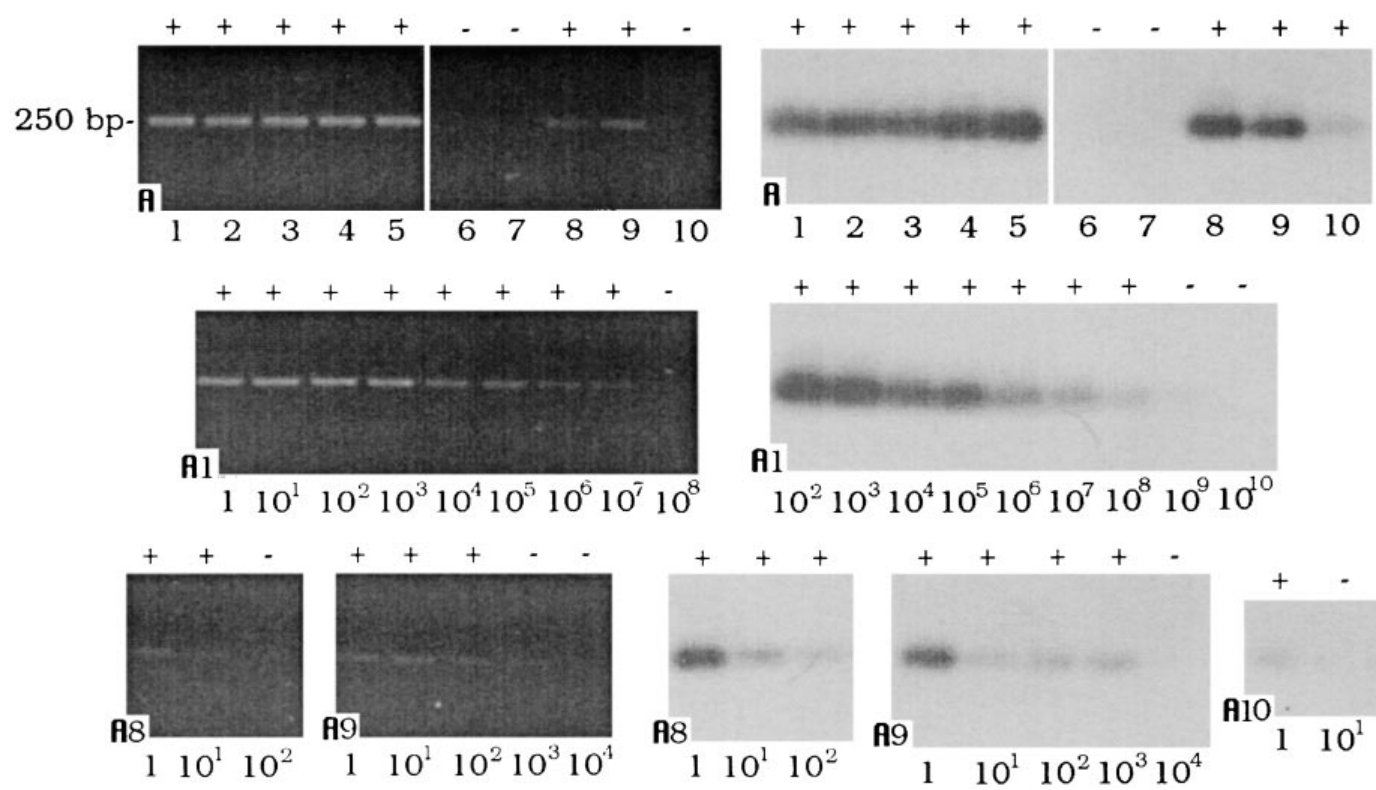

ค1

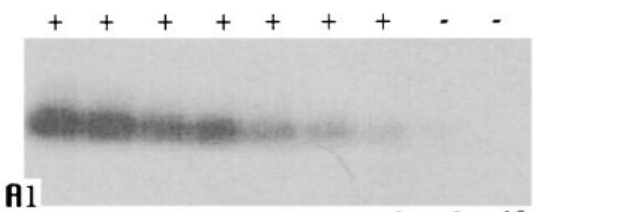

$10^{2} 10^{3} 10^{4} 10^{5} 10^{6} 10^{7} 10^{8} 10^{9} 10^{10}$

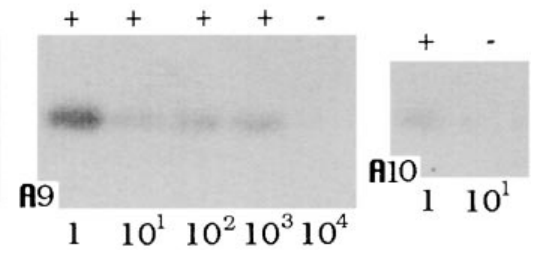

Figure 2. aoHGE-specific DNA amplified from mice challenged with aoHGE (data in Table II, experiment 2). Ethidium bromide staining (left) or Southern blot using a $16 \mathrm{~S}$ rDNA probe (right). (A) Mice administered control (lanes 1-5) or aoHGE antisera (lanes 6-10). ( $A 1, A 8, A 9$, and A10) Serial dilution PCR to quantitate aoHGE. 10-fold dilutions of blood from an aoHGE-infected, control mouse ( $A$, lane 1 ) or aoHGE-infected, experimental mice $(A$, lanes $8-10) .5 \mu \mathrm{l}$ of blood, prepared as described in Methods, was used as the initial aliquot. For example, $A 1\left(10^{8}\right)$ represents a $10^{8}$ dilution of $5 \mu \mathrm{l}$ of blood from the mouse depicted in $A$, lane 1 .
In cases where aoHGE immunization did not fully prevent infection (based on PCR), the number of aoHGE organisms was markedly reduced when compared with infected, control mice, due to the effect of aoHGE-specific antibodies. Conversely, the presence of residual aoHGE DNA in the host, from aoHGE that had been injected into the host during tick-borne transmission, resulted in PCR positivity. In either case, morulae visualization, culture, and PCR data indicate that aoHGE vaccination affords substantial protection against tick-transmitted challenge, and that PCR is the most sensitive method of detection.

Ehrlichia are obligate intracellular pathogens related to Rickettsia, Coxiella, and Chlamydia (20). In general, cellular immune responses are necessary for effective immunity to these groups of microbes, and for that matter, against gramnegative intracellular bacteria such as Listeria (21-27). These data show that it is possible to elicit partial immunity against aoHGE in an experimental murine model. Furthermore, the protective capacity of vaccination with aoHGE lysates extended to tick-borne infection, the natural mode of disease transmission, and passive administration of aoHGE antisera were sufficient for substantial protection. Effective humoral immunity against aoHGE challenge suggests that aoHGE may reside, albeit transiently, in the extracellular environment during part of its life cycle: perhaps in the bloodstream as the pathogen moves among neutrophils, or as the microbe migrates from the tick to the mammalian host. Antibodies could potentially interact directly with aoHGE to facilitate complement-mediated lysis or opsonization at either of these intervals. Now that it is possible to induce immunity against ticktransmitted aoHGE in a model system, the specific antigens that elicit protective antibodies can be identified, the duration of long-term immunity studied, and the mechanisms by which humoral responses afford substantial protection can be elucidated.

\section{Acknowledgments}

This study was supported by National Institutes of Health grant A141440. W. Sun is a postdoctoral fellow of the American Heart Association. J.W. IJdo is a Daland Fellow of the American Philosophical Society and a postdoctoral fellow of the Markey Foundation.

\section{References}

1. Walker, D.H., A.G. Barbour, J.H. Oliver, R.S. Lane, J.S. Dumler, D.T. Dennis, D.H. Persing, A.F. Azad, and E. McSweegan. 1996. Emerging bacterial zoonotic and vector-borne diseases. Ecological and epidemiological factors. JAMA (J. Am. Med. Assoc.). 275:463-469.

2. Bakken, J.S., J.S. Dumler, S.M. Chen, M.R. Eckman, L.L. Van Etta, and D.H. Walker. 1994. Human granulocytic ehrlichiosis in the upper Midwest United States. A new species emerging? JAMA (J. Am. Med. Assoc.). 272:212-218. 3. Bakken, J.S., J. Krueth, C. Wilson-Nordskog, R.L. Tilden, K. Asanovich, and J.S. Dumler. 1996. Clinical and laboratory characteristics of human granulocytic ehrlichiosis. JAMA (J. Am. Med. Assoc.). 275:199-205.

4. Dumler, J.S., and J.S. Bakken. 1995. Ehrlichial diseases of humans: emerging tick-borne infections. Clin. Infect. Dis. 20:1102-1110.

5. Dumler, J.S., and J.S. Bakken. 1996. Human granulocytic ehrlichiosis in Wisconsin and Minnesota: a frequent infection with the potential for persistence. J. Infect. Dis. 173:1027-1030.

6. Pancholi, P., C.P. Kolbert, P.D. Mitchell, K.D. Reed, Jr., J.S. Dumler, J.S. Bakken, S.R. Telford, and D.H. Persing. 1995. Ixodes dammini as a potential vector of human granulocytic ehrlichiosis. J. Infect. Dis. 172:1007-1012.

7. Hardalo, C.J., V. Quagliarello, and J.S. Dumler. 1995. Human granulocytic ehrlichiosis in Connecticut: report of a fatal case. Clin. Infect. Dis. 21:910-914.

8. Walker, D.H., and J.S. Dumler. 1996. Emergence of the ehrlichiosis as human health problems. J. Emerg. Infect. Dis. 2:18-29.

9. Greig, B., K.M. Asanovich, P.J. Armstrong, and J.S. Dumler. 1996. Geographic, clinical, serologic, and molecular evidence of granulocytic ehrlichiosis, a likely zoonotic disease, in Minnesota and Wisconsin dogs. J. Clin. Microbiol. 34:44-48.

10. Chen, S.M., J.S. Dumler, J.S. Bakken, and D.H. Walker. 1994. Identification of a granulocytotropic Ehrlichia species as the etiologic agent of human 
disease. J. Clin. Microbiol. 32:589-595.

11. Woldehiwet, Z. 1983. Tick-borne fever: a review. Vet. Res. Commun. 6: $163-175$

12. Magnarelli, L.A., K.C. Stafford, T.N. Mather, M.T. Yeh, K.D. Horn, and J.S. Dumler. 1995. Hemocytic rickettsia-like organisms in ticks: serologic reactivity with antisera to Ehrlichiae and detection of DNA of agent of human granulocytic ehrlichiosis by PCR. J. Clin. Microbiol. 33:2710-2714.

13. Telford, S.R., J.E. Dawson, P. Katavolos, C.K. Warner, C.P. Kolbert, and D.H. Persing. 1996. Perpetuation of the agent of human granulocytic ehrlichiosis in a deer tick-rodent cycle. Proc. Natl. Acad. Sci. USA. 93:6209-6214.

14. Magnarelli, L.A., J.S. Dumler, J.F. Anderson, R.C. Johnson, and E. Fikrig. 1995. Coexistence of antibodies to tick-borne pathogens of babesiosis, ehrlichiosis, and Lyme borreliosis in human sera. J. Clin. Microbiol. 33:30543057 .

15. Green, M.C. 1989. Catalog of mutant genes and polymorphic loci. In Genetic Variants and Strains of the Laboratory Mouse. 2nd ed. M.F. Lyon and A.G. Searle, editors. Oxford University Press, New York. 12-404.

16. Telford, S.R., T.J. Lepore, P. Snow, C.K. Warner, and J.E. Dawson. 1995. Human granulocytic ehrlichiosis in Massachusetts. Ann. Intern. Med. 123: $277-279$.

17. Goodman, J.L., C. Nelson, B. Vitale, J.E. Madigan, J.S. Dumler, T.J. Kurtti, and U.G. Munderloh. 1996. Direct cultivation of the causative agent of human granulocytic ehrlichiosis. N. Engl. J. Med. 334:209-215.

18. Hanson, B.A., C.L. Wisseman, A. Waddell, and D.J. Silverman. 1981. Some characteristics of heavy and light bands of Rickettsia prowazekii on renografin gradients. Infect. Immun. 34:596-604.

19. Barlough, J.E., J.E. Madigan, E. DeRock, J.S. Dumler, and J.S. Bakken. 1995. Protection against Ehrlichia equi is conferred by prior infection with the human granulocytotropic Ehrlichia (HGE agent). J. Clin. Microbiol. 33:33333334 .

20. Roux, V., and D. Raoult. 1995. Phylogenetic analysis of the genus Rickettsia by 16 S rDNA sequencing. Res. Microbiol. 146:385-396.

21. Zhang, Y.X., N. Zhi, S.R. Yu, Q.J. Li, G.Q. Yu, and X. Zhang. 1995. Protective immunity induced by $67 \mathrm{kD}$ outer membrane protein of phase I Coxiella burnetii in mice and guinea pigs. Acta Virol. 38:327-332.

22. An, L.L., E. Pamer, and J.L. Whitton. 1996. A recombinant minigene vaccine containing a nonameric cytotoxic-T-lymphocyte epitope confers limited protection against Listeria monocytogenes infection. Infect. Immun. 64:16851693.

23. Dumler, J.S., C.L. Wisseman, Jr., P. Fiset, and M.L. Clements. 1992 Cell-mediated immune responses of adults to vaccination, challenge with Rickettsia rickettsii, or both. Am. J. Trop. Med. Hyg. 46:105-115.

24. Hickman, C.J., C.K. Stover, S.W. Joseph, and E.V. Oaks. 1993. Murine T-cell response to native and recombinant protein antigens of Rickettsia tsutsugamushi. Infect. Immun. 61:1674-1681.

25. Knight, S.C., S. Iqball, C. Woods, A. Stagg, M.E. Ward, and M. Tuffrey. 1995. A peptide of Chlamydia trachomatis shown to be a primary T-cell epitope in vitro induces cell-mediated immunity in vivo. Immunology. 85:8-15.

26. Miller, M.A., M.J. Skeen, and H.K. Ziegler. 1995. Nonviable bacterial antigens administered with IL-12 generate antigen-specific $\mathrm{T}$ cell responses and protective immunity against Listeria monocytogenes. J. Immunol. 155:48174828 .

27. Szalay, G., C.H. Ladel, and S.H. Kaufmann. 1995. Stimulation of protective CD8+ T lymphocytes by vaccination with nonliving bacteria. Proc. Natl. Acad. Sci. USA. 92:12389-12392. 